\title{
A METHOD FOR POLISHED LENSES INSPECTION
}

\author{
Dana Rizescu ${ }^{1}$, Ciprian Ion Rizescu ${ }^{1}$ \\ 1 University POLITEHNICA of Bucharest, Mechatronics and Precision Mechanics Department \\ Faculty of Mechanical Engineering, Bucharest, Romania \\ Email: ciprian.rizescu@upb.ro
}

\begin{abstract}
The aim of this paper is focused to determine the final optical behaviour of a lens or a couple of them glued or not by their response in a telecentric beam light. Due to a computer analyze based on determining the spectral curve response by spectrometry and a simulation program, the lens manufacturer can decide either the ensemble is good or not for its purpose or application. The decision to replace the group of lenses and to remanufacture it or not is up to manufacturer but suggested by computer program because the simulation gives a clue of what could be the main cause of damage (the thickness of glue, displacing the lenses or the wrong pairing). The simulation was developed in Matlab environment. The interface allow the users to select any image plane position. The inspection process is very complex for three reasons: delicate process, higher expected quality and transferred responsibility. This is why the authors need to rethink the inspection.
\end{abstract}

Keywords: Lens , Intelligent Control, Spectrometry, Transmittance, Spectral Curve.

\section{Introduction}

The authors experienced a lot of quality images criteria to say if a lens or group of them is good or not for a certain application. Many of those criteria are imposed by beneficiaries and meet their needs. The authors believe that spectral response can be one decisive factor which can help manufacturer to figure out how images will be and, at the same time, to have an answer if the decision is not favourable for lens or group of lenses: is that for recycle bin or for garbage. Spectral response of real lens or a pair of them is transferred to computer from a spectrometer via special card.

The image of real spectral curve (fig. 1) is compared with the simulated one (fig. 5) [1]. Before any response, the manufacturer set up the major differences accepted for individuals, regarding dimensions or glass characteristics. The automatic response displays as many results as the user want to. For interest is focal distance, mainly, because it can be related to any normal or wrong behaviour. But there are other criteria too, (such as ghost images or low quality images) which can induce a decision. If that two images are matching, the answer is (GOOD) and the lens or group of lenses can proceed; if those two images don't match, the computer changes by simulation, the parameters using the limits imposed, to bring the simulated image as close as it can be to real one. The new parameters are to be displayed for manufacturer which can decide if lens or group of lenses can be used in other application as a whole, or can be re- manufactured one by one, both of them or only one, or neither nor [2].

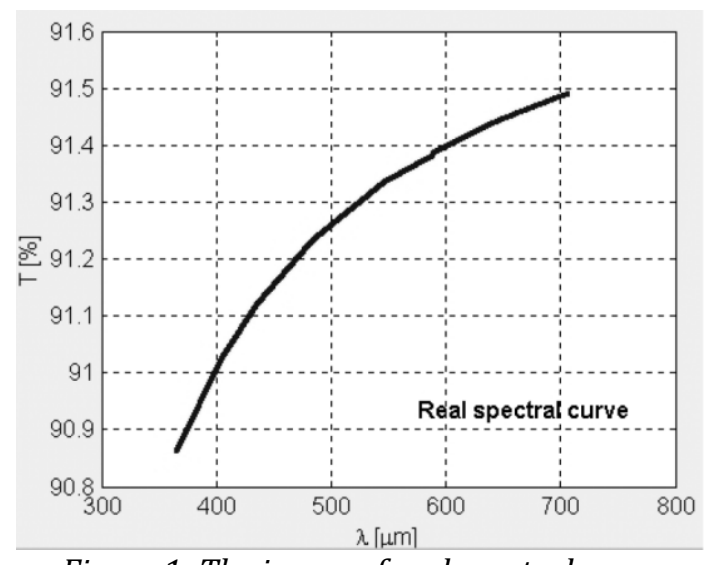

Figure 1: The image of real spectral curve

The figure 2 shows the optical layout of a positive lens; it emphasizes the configuration of second order images caused by internal multiple reflections. At the same time, the overall transmission is diminished for all spectral length or only for extreme one. The behaviour is showed in the simulation program and the user can "see" the differences. All those differences are compared with the limits and the final answer is based on this comparison. The authors use this method to suggest how is ray tracing appear in the real behaviour. A matrix giving a spatial pattern as it is figured in figure 3 crosses the lens. This pattern allows manufacturer to be very close to real image and to understand if the lens can be good for any application or not. 
Transmittance of the positive lens dependant on the height of incidence (A 3D representation which was obtained by considering a distribution of the rays of the incident parallel beam in the entrance pupil.



Figure 2: The Optical layout of a positive lens



Figure 3: Spatial pattern matrix

The transmittance curves are computed and compared automatically. The answer GOOD or WRONG is just a clue for manufacturer. $\mathrm{He}$ is thinking about what can be the cause of wrong behaviour makes the final decision. If the lenses work as a group (glued or not) the manufacturer can have the simulated behaviour of each one and the decision can be more accurate.

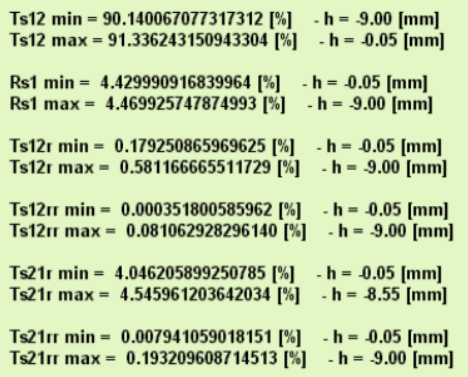

Figure 4 shows simulated curves and figure 5 presents the spectral curve, captured via computer card [3].

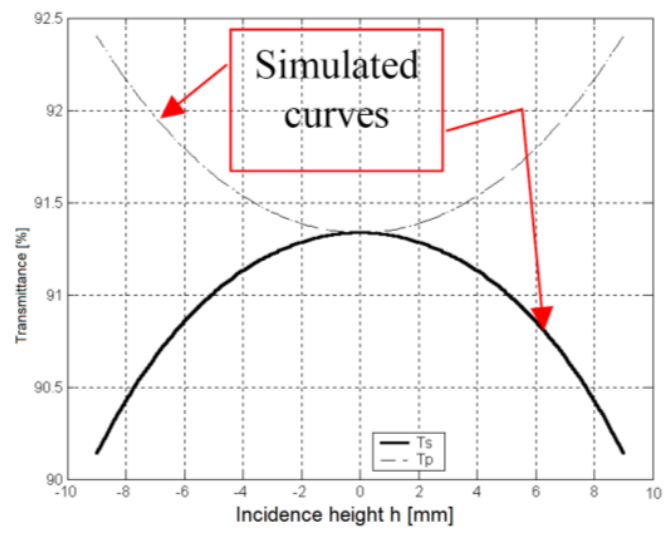

Figure 4: Simulated curves



Figure 5: Spectral characteristic of the positive lens in the visible spectrum

Transmittance of the positive lens dependant on the height of incidence (it was computed using Fresnel's coefficients. Finally, numerical result makes the decision easier and accurate as it is shown in figure 6. These numerical results of the spectra-energetic characteristics: reflectance/transmittance are shown not only of the primary image but also for the secondary images produced by internal reflections

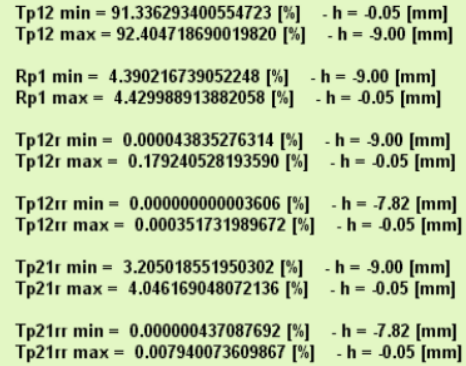

Figure 6: Numerical results of the spectra-energetic characteristics

\section{Validation of the Method}

The authors faced a lot of questions regarding the influence of the optical and dimensional parameters on the transmittance curve.
That's why, the next step was to display these influences. As it is shown in fig. 7 the curves for transmittance have different shapes linked with the deviations suffered of some basic parameters for interest in this application. 


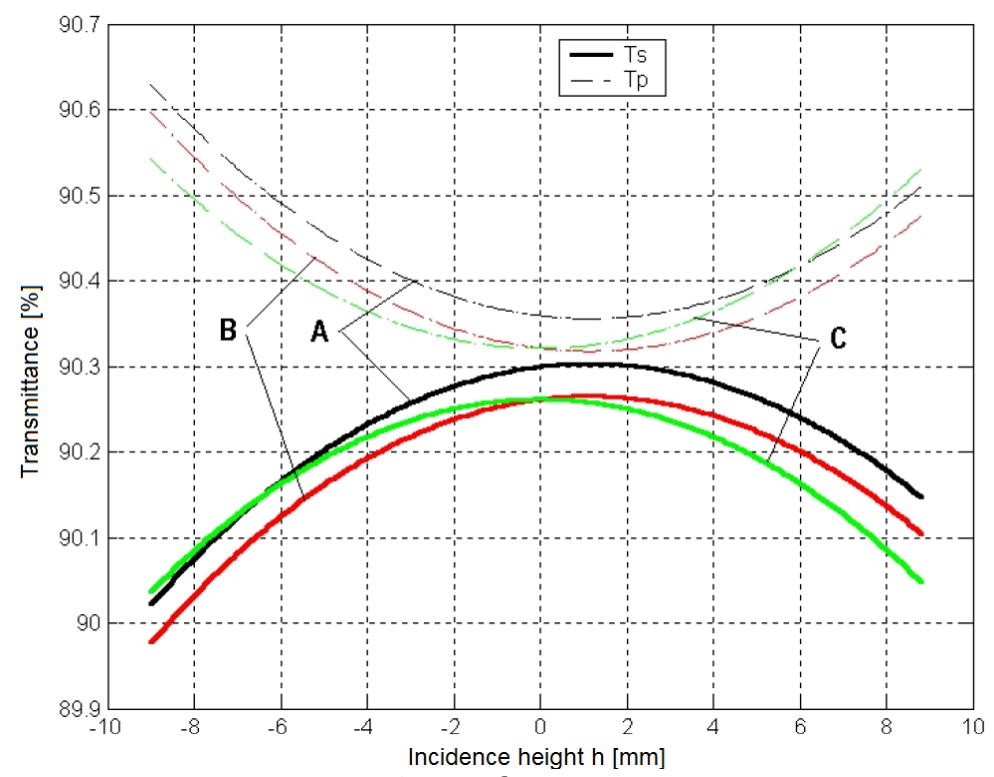

Figure 7: Curves for transmittance

A - identical curve for both lenses on the gluing surface ;

$\mathrm{B}$ - the radius $\mathrm{R} 2>\mathrm{R} 3$, causing an interlaceless at the center;

$\mathrm{C}$ - the radius $\mathrm{R} 2<\mathrm{R} 3$, causing an interlaceless at the margins.

The curves A, B, C show the transmittance for different values of radius R1, R2, R3, R4 of a group of glued lenses. The condition of gluing is to have the same nominal values for gluing radius (in our case R2 and R3). In reality, the limits admitted for nominal values and randomly paired cause a third lens (very thin one, made from glue) but with significant influence in total transmission [4]. The percentage of losing transmitted light varies up to $0.1 \%$. So, the spectrometer has to be of the first class accuracy to have such accurate measures.

The behaviour for negative lenses is pretty similar. In figure 8 is shown the optical layout of negative lens.

The major difference between those two types of lenses is the ghost images which, in most cases is virtual one. Due to this position, the image can cause a chain of ghost images, if negative lens is coupled with a positive one.

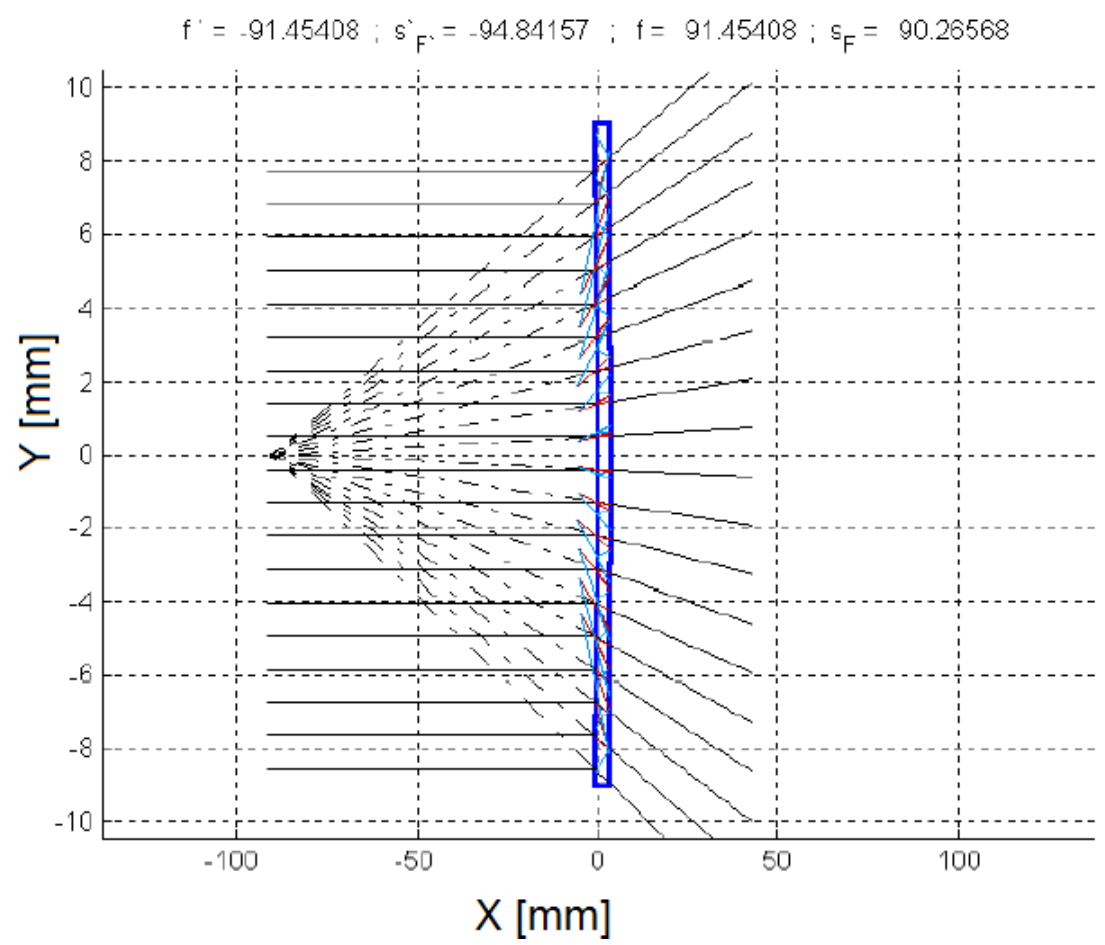

Figure 8: Optical layout for negative lens 


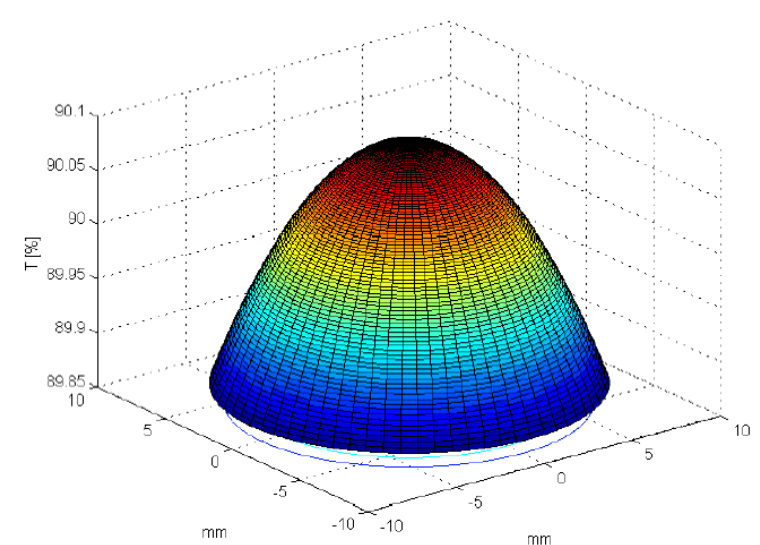

Figure 9: Inspection matrix

In figure 9 is presented the inspection matrix.

Optical layout for a doublet consisting of the positive and negative lenses studied above and separately are shown in figure 10 .



Figure 10: Optical layout for a doublet

The behaviour for coupled lenses (usually glued) is shown in the figure 11 . The ghost images are neglected in this picture due to overloading it. The influence of those ghost images is figured out in figure 12 .

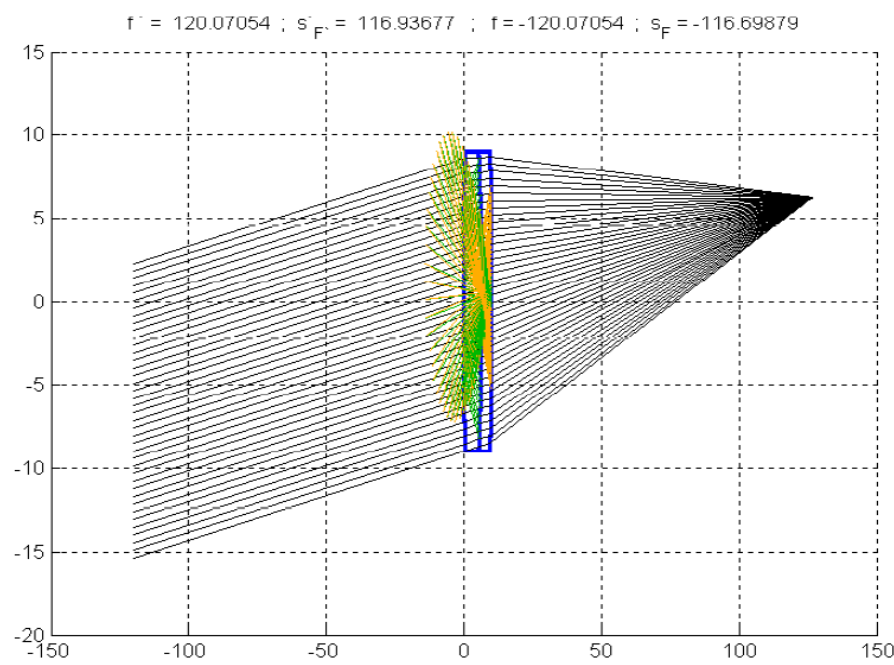

Figure 11: Behaviour of coupled lenses

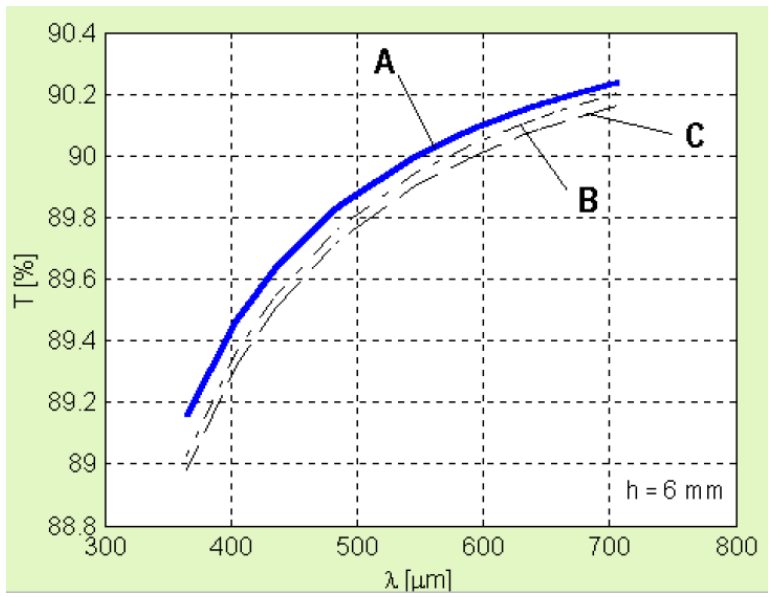

Figure 12: Influence of ghost images

The differences between behaviour of one component and the couple as a whole can be seen comparing the figure 12 with figure $5[5,6]$.
The transmission of coupled is lower (T 90\% at $500 \mathrm{~nm}$.) then of each component. The major influence, except the thickness of lenses is due to ghost images. Figure 13 represents transmission of coupled lenses for a distribution in the entrance pupil. The numerical results for spectra-energetic characteristics are shown in figure 14.

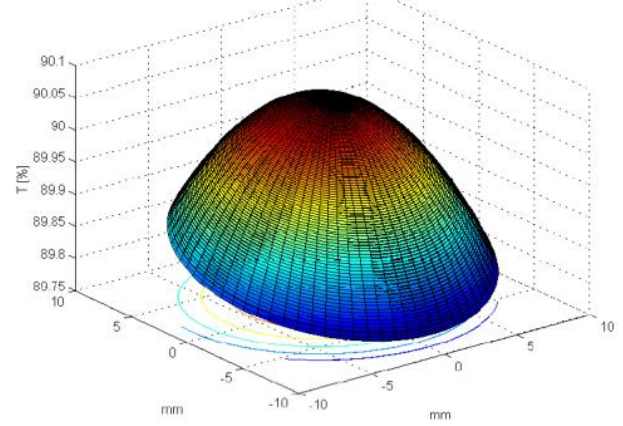

Figure 13: Transmission of coupled lenses 


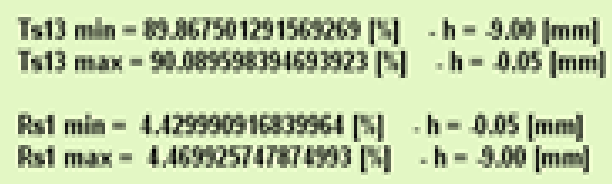

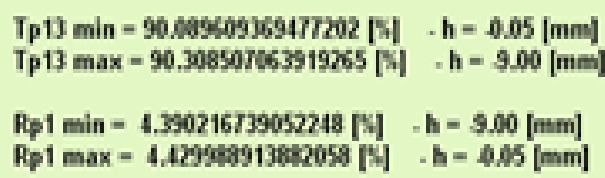

Figure 14: Numerical results of the spectra-energetic characteristics for a doublet

\section{Simulation Interface}

The simulation program was created in the MATLAB environment and offers the following possibilities:

- input the geometric characteristics of lenses and doublets , cemented or air-spaced (accumulation radius, thickness, useful and total diameter).

- choose an optical material for a certain wavelength or a spectral field from a data base;

- determination of the paraxial characteristics of the optical system;

- determination of the spectra-energetic characteristics;

- shows the formation of the parasitic ("ghost") images due to multiple internal reflections
- highlight the influence of the geometric parameters variation on the spectral characteristic

The Matlab interface has five options according to the design conditions:

1 = optical scheme;

$2=$ the image in a plane;

3 = spot diagram;

$4=$ transmittance;

5 = spectral characteristic;

0 = stop;

The interface allow the users to select any image plane position. There were selected different image plane positions (L). Some simulation results are presented in figures 15, 16, 17, 18, 20, 21, 22 and 23 as it follows:

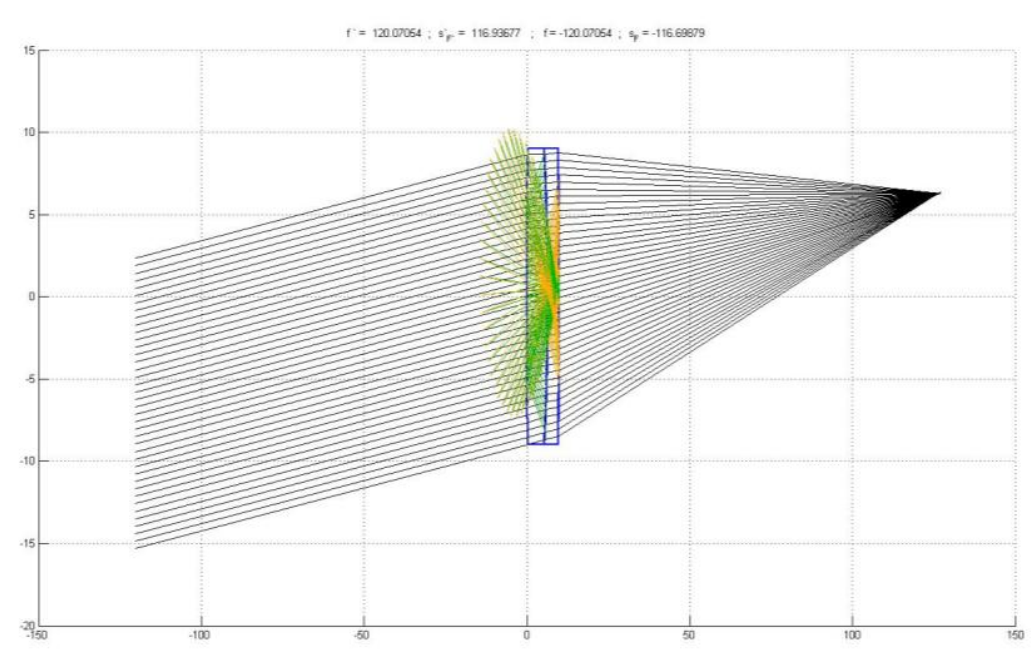

Figure 15: Optical scheme

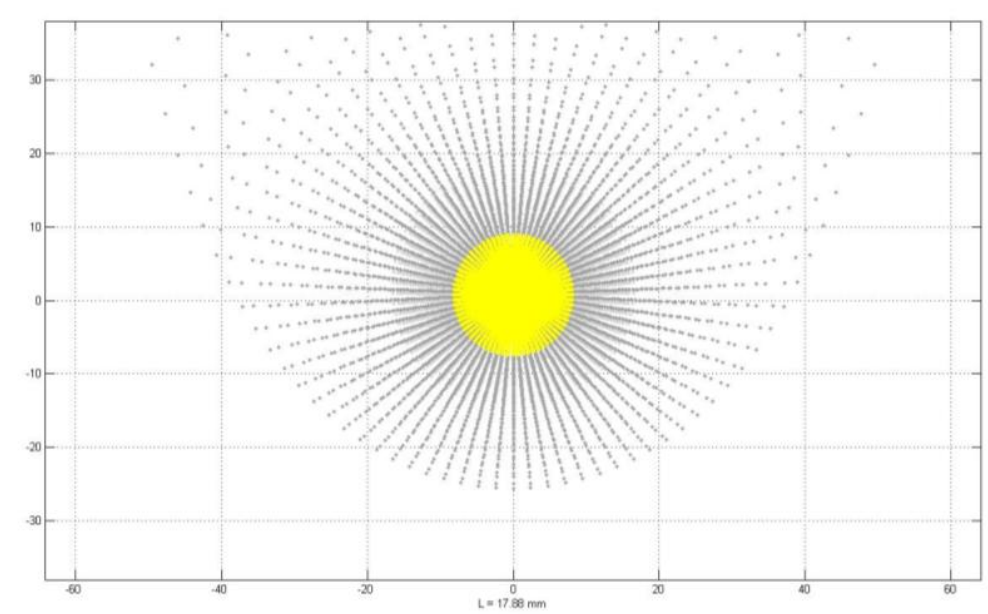

Figure 16: The image plane for $L=17.88 \mathrm{~mm}$ 


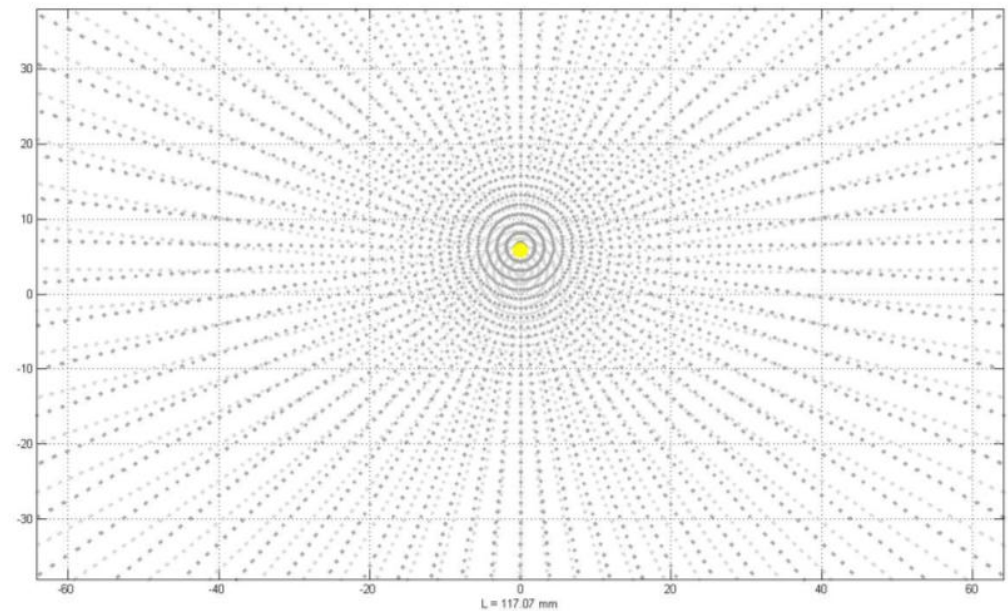

Figure 17: The image plane for $L=117.07 \mathrm{~mm}$

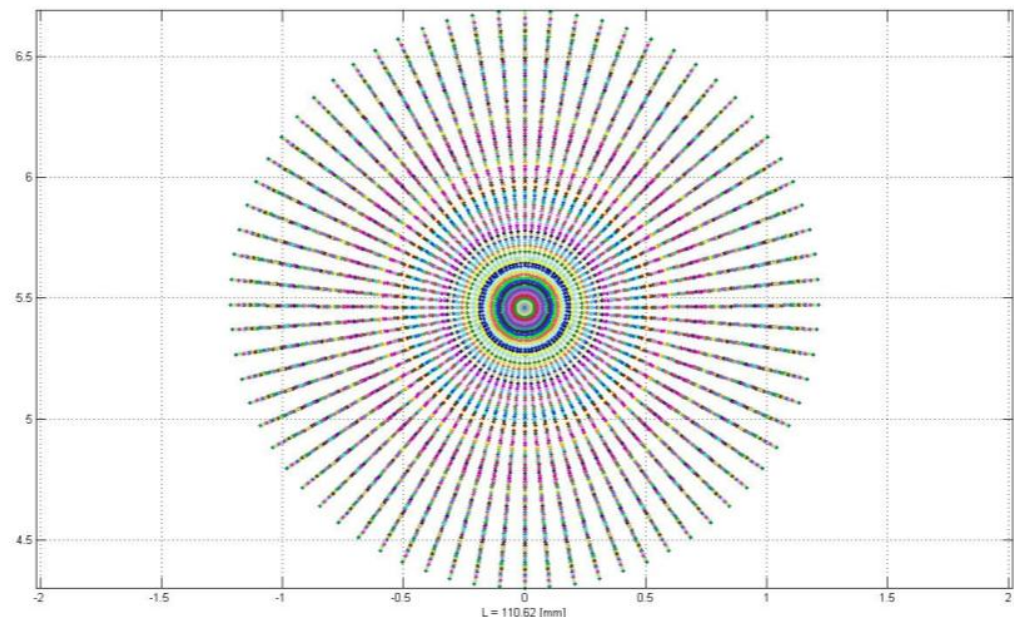

Figure 18: Spot diagram for $L=110.62 \mathrm{~mm}$

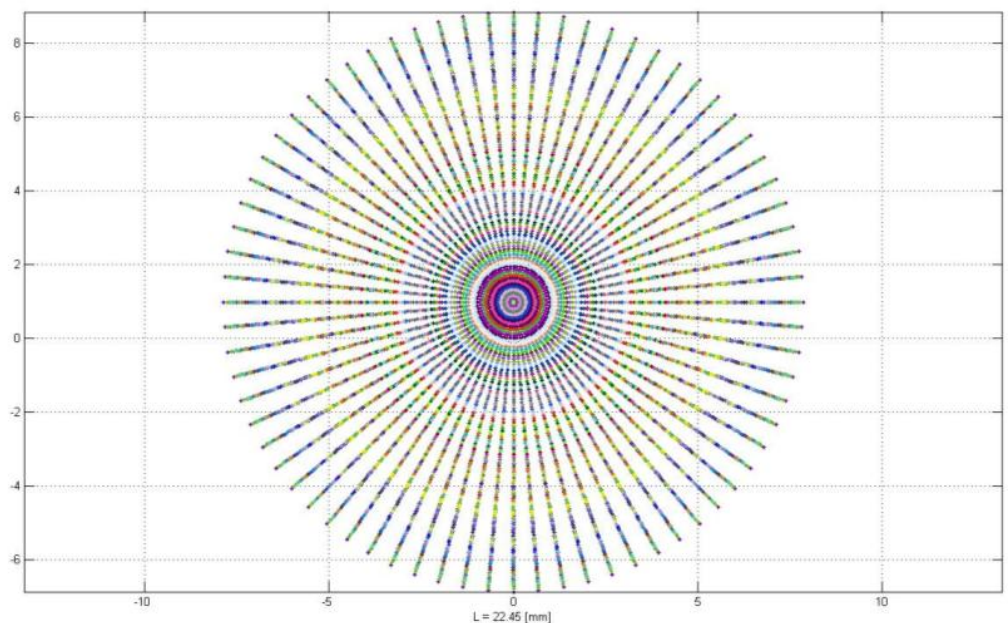

Figure 19: Spot diagram for $L=22.45 \mathrm{~mm}$

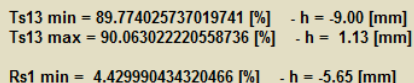

$T p 13 \mathrm{~min}=90.116135689103757[\%] \quad-\mathrm{h}=1.13[\mathrm{~mm}]$


Tp13 $\max =90.397047987475972[\%] \quad-h=-9.00[\mathrm{~mm}$ $\begin{array}{ll}\text { Rs1 } \min =4.429990434320466[\%] & -h=-5.65[\mathrm{~mm}] \\ \text { Rs1 } \max =4.537092573504129[\%] & -\mathrm{h}=9.00[\mathrm{~mm}]\end{array}$

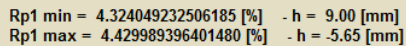

Figure 20: Numerical results for transmittance 


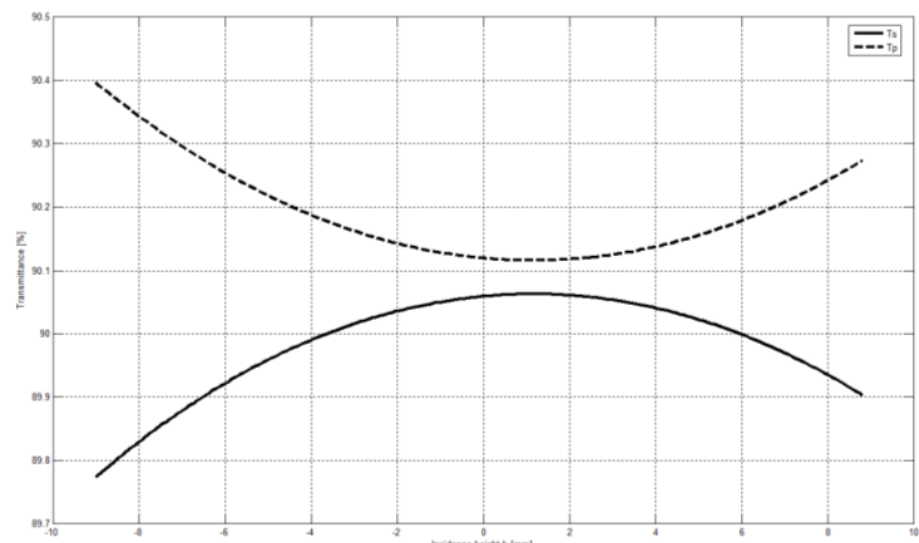

Figure 21: Transmittance

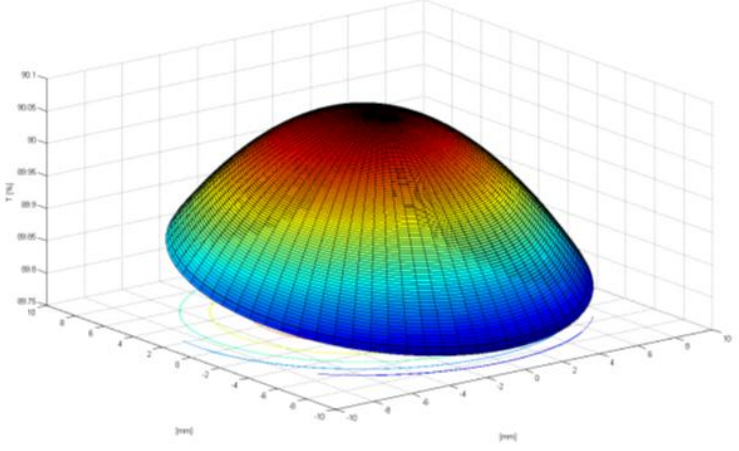

Figure 22: Transmittance - 3D representation

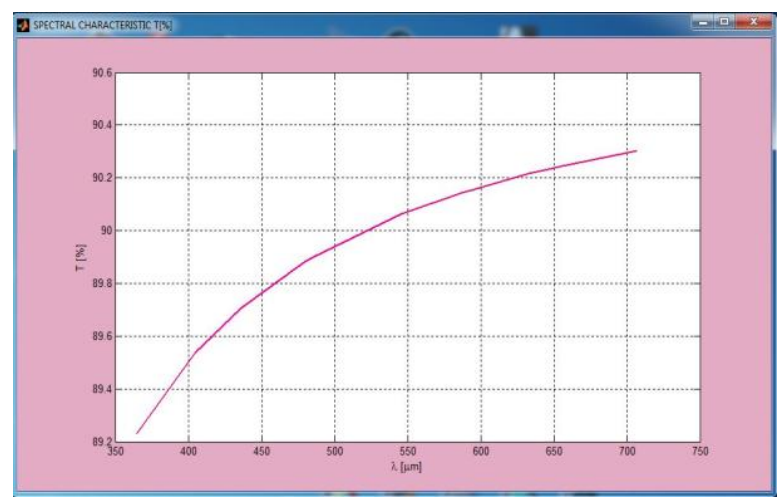

Figure 23: Spectral characteristic

\section{Conclusions}

Intelligent inspection based on comparing spectral curves (simulated and acquainted) can give an answer about how would be the behaviour of coupled lenses as a whole in the future ensemble. The authors developed a Matlab interface to study different optic components and their spectral response.

\section{References}

[1] G.C. Moisil, E. Curatu, Optica : Teorie și Aplicații, Ed. Tehnică, 1986, pp. 79-87, 95.

[2] A.Ov.Trufasu, Noțiuni fundamentale aplicate în optica tehnică, Ed. Bren 77, 2000, pp.34-36, 9498, 2000.

[3] Subasri, R., Malathi, R., Jyothirmayi, A., Hebalkar, N.Y., Ceramics International, 38, no. 7, 2012, p. 5731.

[4] Samson, F., Surface Coating Technology, 81, 1996, p. 79.

[5] Szeghalmi, A., Helgert, M., Brunner, R., Heyroth, F., Gosele, U., Knezl, M., Applied Optics, 48, 2009, p. 1727.

[6] Hunt S, Cuthill IC, Bennett ATD, Church SC, Partridge JC. 2001. Is the ultraviolet waveband a special communication channel in avian mate choice? , J. Exp. Biol. 204, 2499-2507. 